\title{
Towards a Threat Model for Provenance in e-Science
}

\author{
Luiz M.R. Gadelha Jr. ${ }^{1}$, Marta Mattoso ${ }^{1}$, Michael Wilde ${ }^{2}$, and Ian Foster ${ }^{2}$ \\ 1 Computer and Systems Engineering Program \\ Federal University of Rio de Janeiro, Brazil \\ \{gadelha, marta\}acos.ufrj.br \\ ${ }^{2}$ Computation Institute \\ University of Chicago / Argonne National Laboratory, USA \\ \{wilde, foster\}amcs.anl.gov
}

\begin{abstract}
Scientists increasingly rely on workflow management systems to perform large-scale computational scientific experiments. These systems often collect provenance information that is useful in the analysis and reproduction of such experiments. On the other hand, this provenance data may be exposed to security threats which can result, for instance, in compromising the analysis of these experiments, or in illegitimate claims of attribution. In this work, we describe our ongoing work to trace security requirements for provenance systems in the context of e-Science, and propose some security controls to fulfill them.
\end{abstract}

\section{Introduction}

As an important paradigm of scientific research, computer simulations are increasingly being used to perform computational scientific experiments. As the scale of these experiments increase, scientific workflow management systems become a relevant tool to specify, execute, and analyze them. These systems can collect provenance information, often distributed in grids or remote clusters, that is useful in the analysis and reproduction of such experiments. If the appropriate security controls are not in place, provenance systems may be exposed to threats that may compromise the integrity, confidentiality, or availability of provenance data. In this work, we describe our ongoing work to trace security requirements for provenance systems in the context of e-Science, and propose some security controls to fulfill them. The study of security issues in provenance systems is relatively recent. However, some important security requirements, described in section 2 were not yet identified in related academic works, to our knowledge. In section 3, we conclude describing subsequent steps in our provenance security research.

\section{Security Requirements for Provenance Systems}

The typical execution of a workflow involves specifying its flow using some mechanism, such as a parallel scripting language or a GUI-based workflow specification tool. Later on, it can be executed by a workflow management system, this involves selecting appropriate computational resources, submitting tasks to these resources, and transferring data. After the experiment is executed, scientists typically face the challenge of analyzing a large number of output data files to understand the outcome of the

D.L. McGuinness, J.R. Michaelis, and L. Moreau (Eds.): IPAW 2010, LNCS 6378, pp. 277-279, 2010.

(C) Springer-Verlag Berlin Heidelberg 2010 
experiment, provenance systems are useful in this context since they can help to determine, for instance, which tasks where executed to generate a particular data object, and which parameters were used for these tasks. This provenance data is usually collected and stored during workflow execution, to describe causal relationships between tasks and data (retrospective provenance); or during workflow specification, to describe the planned tasks, and data flow (prospective provenance). In general, provenance data is accessed and analyzed by scientists using a query language, such as SQL. In our ongoing threat modeling effort, we are enumerating threats to each of these components of a provenance system. Many of these are already taken into account by security frameworks for underlying technologies used by provenance systems, such as databases and grids. Provenance security is a relatively recent research issue [12] [7] [14], found in different areas such as scientific workflows, databases, and storage systems. Provenance data is useful in security audits [1], and there are cases in which the subject of provenance data may lead to privacy concerns [3]. Braun et al. [2] analyze the problem of providing adequate access control techniques to provenance data, observing that it describes causal relationships, that are not adequately protected by commonly used access control techniques. Hasan et al. [9] [8] propose a security solution with the goal of protecting confidentiality and integrity of provenance data. They use asymmetric cryptography for achieving confidentiality and signature-based checksums for achieving integrity. Nagappan et al. [10] present a model for sharing provenance data that uses role-based access control techniques where the user dynamically select its confidentiality level. A common approach for protecting provenance information [2] [10] [11] is to use access control mechanisms to prevent unauthorized access to this information. None of these approaches allow for non-restricted dissemination of provenance information with maintenance of correct attribution, a requirement in the context of e-Science. Scientists, specially in the life sciences, often avoid sharing details of experiments prior to publishing their results in some academic journal or event, to assure correct attribution of scientific results. During this interval, scientific collaboration is prevented. Therefore, security controls that prevent illegitimate claims of attribution are an important security requirement for provenance systems. These controls must allow the verification of not only who executed an experiment but also when it was executed. A combination of digital signatures and cryptographic timestamps [6] were used in the Kairos [5] security architecture for provenance systems to provide these properties. Another desirable security property is fine-grained access control, where scientists can delegate to their collaborators access to provenance data, so it can be read or modified.

\section{Concluding Remarks}

This work describes our progress in defining a threat model and proposing security controls for provenance systems in the context of e-Science. We identify the assurance of correct attribution of scientific results as an important security requirement for these systems. For this purpose, we proposed Kairos [5], a security architecture for provenance that uses cryptographic timestamps [6] and digital signatures. We are working with the Swift [13] parallel scripting system to extend its provenance system [4] with appropriate security controls. As future work, we will investigate fine-grained access control techniques, and a data model to store and query security properties. 


\section{Acknowledgement}

This work was supported in part by CAPES, CNPq, NSF grant OCI-0944332, and the Office of Advanced Scientific Computing Research, Office of Science, U.S. Department of Energy, under Contract DE-AC02-06CH11357.

\section{References}

1. Aldeco-Pérez, R., Moreau, L.: Provenance-based Auditing of Private Data Use. In: Proc. of the BCS International Academic Research Conference, Visions of Computer Science (2008)

2. Braun, U., Shinnar, A., Seltzer, M.: Securing Provenance. In: Proc. 3rd USENIX Workshop on Hot Topics in Security, HotSec 2008 (2008)

3. Davidson, S., Khanna, S., Roy, S., Cohen-Boulakia, S.: Privacy Issues in Scientific Workflow Provenance. In: Proceedings of the 1st International Workshop on Workflow Approaches to New Data-centric Science, WANDS 2010 (2010)

4. Gadelha, L., Clifford, B., Mattoso, M., Wilde, M., Foster, I.: Provenance Management in Swift. Future Generation Computer Systems (2010) (in press, accepted manuscript)

5. Gadelha, L., Mattoso, M.: Kairos: An Architecture for Securing Authorship and Temporal Information of Provenance Data in Grid-Enabled Workflow Management Systems. In: Proc. 4th IEEE International Conference on e-Science (e-Science 2008), pp. 597-602 (2008)

6. Haber, S., Stornetta, W.: How to Time-Stamp a Digital Document. Journal of Cryptology 3(2), 99-111 (1991)

7. Hasan, R., Sion, R., Winslett, M.: Introducing Secure Provenance: Problems and Challenges. In: Proc. 2007 ACM Workshop on Storage Security and Survivability (StorageSS 2009), pp. 13-18 (2007)

8. Hasan, R., Sion, R., Winslett, M.: Preventing history forgery with secure provenance. ACM Transactions on Storage 5(4), 1-43 (2009)

9. Hasan, R., Sion, R., Winslett, M.: The Case of the Fake Picasso: Preventing History Forgery with Secure Provenance. In: Proc. 7th USENIX Conference on File and Storage Technologies (FAST 2009), pp. 1-14 (2009)

10. Nagappan, M., Vouk, M.: A Model for Sharing of Confidential Provenance Information in a Query Based System. In: Freire, J., Koop, D., Moreau, L. (eds.) IPAW 2008. LNCS, vol. 5272, pp. 62-69. Springer, Heidelberg (2008)

11. Ni, Q., Xu, S., Bertino, E., Sandhu, R., Han, W.: An Access Control Language for a General Provenance Model. In: Jonker, W., Petković, M. (eds.) Secure Data Management. LNCS, vol. 5776, pp. 68-88. Springer, Heidelberg (2009)

12. Tan, V., Groth, P., Miles, S., Jiang, S., Munroe, S., Tsasakou, S., Moreau, L.: Security Issues in a SOA-Based Provenance System. In: Moreau, L., Foster, I. (eds.) IPAW 2006. LNCS, vol. 4145, pp. 203-211. Springer, Heidelberg (2006)

13. Wilde, M., Foster, I., Iskra, K., Beckman, P., Espinosa, A., Hategan, M., Clifford, B., Raicu, I.: Parallel Scripting for Applications at the Petascale and Beyond. IEEE Computer 42(11), 50-60 (2009)

14. Xu, S., Ni, Q., Bertino, E., Sandhu, R.: A Characterization of The Problem of Secure Provenance Management. In: Proc. IEEE International Conference on Intelligence and Security Informatics (ISI 2009), pp. 310-314 (2009) 